March-June 2012.

Results 36 patients were identified who were suitable to enter the study, of which 5 declined. 31 patients completed the study (19 females). 17 responded that they will consider $\mathrm{MNH}$ if they became unable to eat or drink. IV and SC route were the most favoured methods, mainly because of familiarity. NG tube feeding was the least favoured route. Most patients felt that maintaining nutrition and hydration was a necessary part of their life. Patient's previous experiences also played a part in their accepting or rejecting $\mathrm{MNH}$.

Conclusions This preliminary study suggests that MNH is likely to be accepted by many patients who may need it near the end of life (although these subjects were not at that stage). It also highlighted the benefit of discussion using visual aids to help patients make an informed choice about having $\mathrm{MNH}$ and the method. We are preparing a larger multicentre study in order to assess whether these findings are replicable in a variety of settings.

\section{P 132 HOW ACCEPTABLE IS MEDICALLY ASSISTED NUTRITION AND HYDRATION IN PATIENTS APPROACHING THE END OF LIFE? - A SERVICE EVALUATION}

Ashique Ahamed, ${ }^{1}$ Sam Fingas, ${ }^{2}$ Adam Beech, ${ }^{3}$ Catherine Nock, ${ }^{3}$ Elizabeth Newell, ${ }^{2}$ Julia Newell, ${ }^{2}$ Sam Ahmedzai ${ }^{3} .{ }^{1}$ Central Manchester University Hospitals NHS foundation Trust, Manchester, United Kingdom; ${ }^{2}$ Sheffield Teaching Hospitals NHS Foundation Trust; ${ }^{3}$ Medcial School, The University of Sheffield

10.1136/bmjspcare-2014-000654.173

Background Patients nearing the end of life can have difficulty in eating and drinking due to variety of reasons. Currently there are differences of opinion in perceived benefits of medically assisted nutrition and hydration $(\mathrm{MNH})$ for dying patients who have inadequate oral intake.

Aims We conducted a study of patients with advancing disease but not yet at end of life, to assess their attitudes towards $\mathrm{MNH}$ if they became unable to have adequate oral intake. A structured interview was developed and piloted before being used. It detailed four different methods of MNH: nasogastric tube feeding, PEG feeding, intravenous drip (IV) including total parenteral nutrition, or subcutaneous hydration (SC). Photographs and samples of the devices were shown. The study was approved as a service evaluation and was conducted in one Sheffield hospital during 